\title{
Metal Transfer Modes in plasma Wire + Arc Additive Manufacture
}

By Sergio Ríos ${ }^{1,2}$, Paul A. Colegrove ${ }^{1}$ and Stewart W. Williams ${ }^{1}$

${ }^{1}$ Welding Engineering and Laser Processing Centre, Cranfield University, University Way, Cranfield, Bedfordshire, UK

${ }^{2}$ Department of Mechanical Engineering, University of Magallanes, Punta Arenas, Región de Magallanes y de la Antártica Chilena, Chile

\begin{abstract}
The stability of Plasma transferred arc Wire + Arc Additive Manufacture is critically dependent on the transfer mode of the wire into the melt pool which can either be in permanent contact (TM1), intermittent (TM2) or fleeting (TM3) and it's governed by the position of the wire. Analysis of the voltage waveforms in conjunction with video imaging was used to understand the preferred mode for process control and how these transfer modes are affected by convex and concave melt pools shapes. The study indicated that stable transfer can be more easily achieved with the convex weld pool, because it minimises the extent of the undesirable TM3 where droplet transfer occurs in an erratic fashion. Furthermore, even though the operational range of TM2 is limited, a control algorithm could be more easily developed for this transfer mode. Finally, video imaging showed how the melt pool oscillation interacts with droplet detachment under some operational conditions.
\end{abstract}

\section{Introduction}

Williams et al. (2016) explains how plasma transferred arc (PTA) is a more suitable option than Gas Metal Arc Welding for Wire + Arc Additive Manufacture (WAAM) of titanium, because it avoids the problem of arc wandering. The ability to maintain stable deposition during PTA-WAAM is essential to making the technology suitable for industrial use, in terms of productivity and process robustness. Stability is dependent upon the metal transfer modes that describe how the filler wire is incorporated into the melt pool. Taguchi et al. $(1979 a, 1979 b)$ found that under most process conditions in PTA and Tungsten Inert Gas (TIG) welding the filler wire is transferred to the work piece by momentarily bridging the gap between melt pool and wire. The relative duration of droplet formation or growth, $t_{g}$ was compared to the time for detachment or contact between the wire and the melt pool, $t_{c}$. This allowed Taguchi et al. (1979a, 1979b) to characterize the metal transfer modes in TIG and PTA welding, into four basic modes:

- Transfer Mode 1 (TM1), with $t_{c} \gg t_{g}$. The wire is in permanent contact with the weld pool, with very occasional interruptions.

- Transfer Mode 2 (TM2), with $t_{c} \approx t_{g}$. The contact lasts for about the same time needed to grow a new droplet.

- Transfer Mode 3 (TM3), with $t_{c}<<t_{g}$. With droplets above a certain size, they grow for much longer and contact when it occurs is swift.

- Transfer Mode 4 (TM4), with $t_{c}=0$. Eventually, contact might be totally impossible under certain conditions. This is observed only under high power conditions in PTA, where the gas velocity in the arc is sufficiently high to tear the droplets off the wire tip. 
When examining the different transfer modes, Yudodibroto et al. (2004) reported that the arc voltage drops during TIG welding, when the droplet becomes a bridge between wire and weld pool. The nuclear industry in Japan made important progress regarding metal transfer mode description and control in TIG and PTA welding, particularly for pipe welding. Imanaga et al. (2000) and Taguchi et al. (1979a, 1979b) used a small DC power supply to pass a control signal of constant current between the wire and the work piece, and measured the voltage drop to indicate when contact with the melt pool occurred. The average contact frequency (CF) was also calculated over a certain period and it was reported that it has a low value in TM1 and TM3 but is maximum in TM2 (depending on the processing conditions). They produced operational envelopes where the transfer modes are indicated for different combinations of wire feed speed, current and plasma gas flow rate. They determined that increasing the power of the process, or the wire height, change the transfer modes towards TM3. Increasing wire feed speed has the opposite effect.

Later on, Kobayashi et al. (1996) observed TM1 to TM3 in pulsed TIG welding, with pulsed wire feeding. They also calculated the relative time of contact, compared to a given window of time. Combining the contact frequency observations and this new indicator, they developed a control system to adjust the wire position. They claimed that the system was successfully used for the automatic repair of pipes in nuclear reactors.

Hagqvist et al. (2014) and Heralić et al. (2012) addressed the same issue with laser-wire deposition in additive manufacture, and developed a control system that was based on keeping the wire in contact with the melt pool at all times. This system detected the increase in resistance of the wire as it "necked" within transfer mode 1 and controlled wire feed speed as compensation. Metal transfer modes in laser processing have also been observed. Dilthey et al. (1995) observed that increasing wire feed speed delayed the formation of the droplet, until the wire had been exposed to enough energy from the arc. This in turned reduced the distance between wire and weld pool, which drastically changed the transfer modes. Miranda et al. (2008) observed similar phenomena in additive manufacture and associated transfer modes and process stability with lased beam diameter and wire position. Seufzer and Taminger (2007) addressed the complexities of low-gravity wire deposition, with electron beam and found that the dominant effect of surface tension kept the growing droplets attached to the wire tip instead of hang towards the molten pool, which narrows the range of operating conditions.

Even though transfer modes have been successfully identified by measuring wire resistance, there is the inconvenience of installing a separate circuit to the process. An alternative is the measurement of arc voltage measurement which is usually already measured and can therefore be used to detect the transfer modes as reported by Yudodibroto (2004). This paper presents a characterisation of the metal transfer modes observed in PTA-WAAM using this second method, which simplifies process monitoring and could potentially be used for automatic control.

\section{Method}

\subsection{Experimental set up}

Figure 1 shows details of the set-up around the torch (1) and the substrate (2). The wire is fed from the side (Figure 2(a)), because it was thought that this might increase the vertical range of suitable wire positions, when the melt pool is concave. Regarding the effect of side feeding on process stability, consider the case of relatively high wire feed speed. In this scenario, the wire melts after a shorter exposure to the arc, compared to low wire feed speed. The exposure may be characterized by the distance $L$ in (Figure 2(a)). Therefore, the parameter $y_{f}$ (Figure 2(a)), which marks the edge between solid and molten wire, would increase with wire feed speed and decrease with arc power. Eventually, the wire may reach the molten pool before being fully melted by the arc. Only then, the molten pool may assist in melting the wire. Thus, the feeding direction may affect process stability only when the pool contributes to melting the wire, which is a sensitive operation condition independently of the wire feeding direction. To adjust the wire position, a positioning unit was developed, including three 
perpendicular micro-metric screws with a positioning accuracy of $0.01 \mathrm{~mm},(4-6)$, and a device for adjusting the angle (3), which was set at $30^{\circ}$ from the horizontal. The whole setup was installed in an argon filled enclosure, where the oxygen concentration was maintained below 2000 ppm.

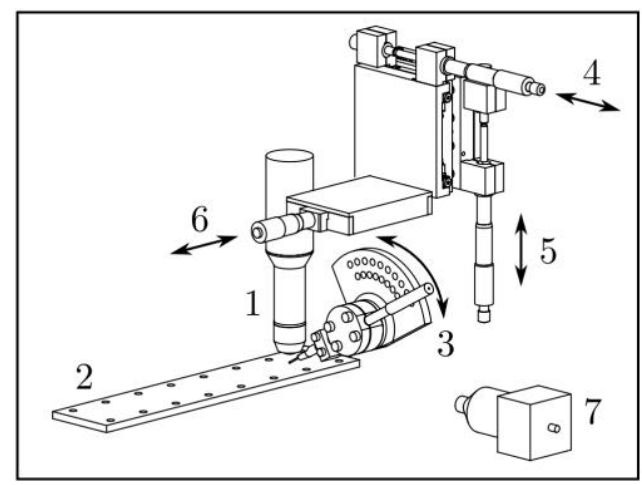

Figure 1 Arrangement of the experimental setup: including the torch (1), the substrate (2), a precision unit for wire positioning (3-6) and the welding video camera (7).

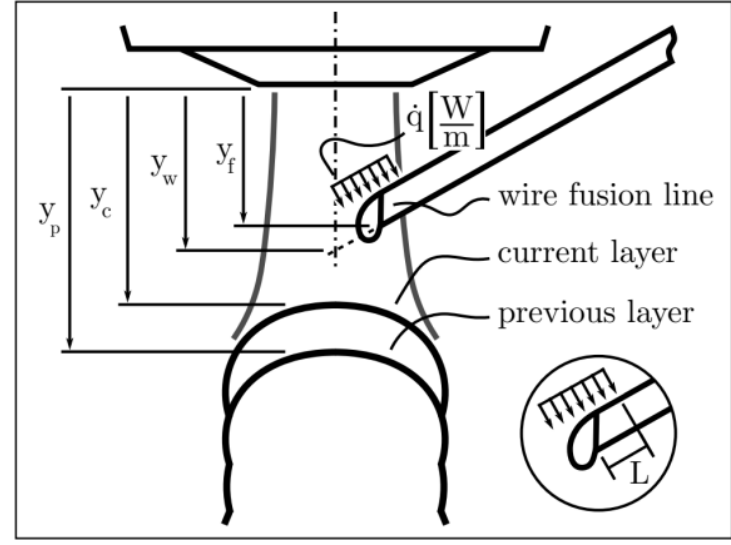

a

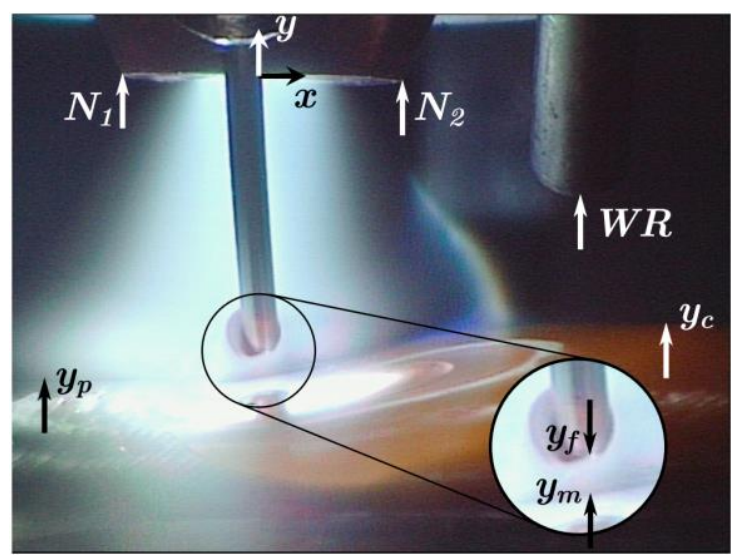

b

Figure 2 (a) Schematic diagram of plasma deposition showing the position of the wire relative to the heat source and deposit; (b) a typical frame indicating the points of interest: nozzle position (N1 and $\mathrm{N} 2)$, the wire reference mark (WR), the previous layer $\left(y_{p}\right)$, current layer $\left(y_{c}\right)$, wire fusion line $\left(y_{f}\right)$, and top of the melt pool $\left(y_{m}\right)$.

\subsection{Welding video}

To visualise the different transfer modes, a welding video camera was installed (Number 7 in Figure 1), which enabled the observation of the process from the side. An example of the view obtained is shown in Figure 2(b), where the labels indicate the points whose $y$ coordinate was measured. These points are: the edges of the nozzle $N 1$ and $N 2$, whose midpoint constitutes the position of the nozzle; the top of the previous layer $y_{p}$ and of the current layer $y_{c}$; the melt pool surface, $y_{m}$; and the wire fusion line, $y_{f}$ which is the lowest visible point along the solid wire.

Notice in Figure $2(a, b)$, that as the wire is fed from the side, it passes between the camera and the torch and it looks like a vertical line. As it is impossible to precisely determine the position of the wire $y_{w}$ on a video frame (such as Figure 2(b)), a tungsten rod attached to the wire feeding tube was used as a wire reference marker and is labelled $W R$. The wire position reference was calibrated to the coordinate of the wire $y_{w}$, with the micrometric adjustment shown in Figure 1. This allowed the relative position of the wire with respect to the nozzle and the melt pool surface to be calculated. The resolution of the camera was $800 \times 600$ pixels and the horizontal field of view was approximately 30 
$\mathrm{mm}$, which makes the resolution about $40 \mu \mathrm{m}$ per pixel. During preliminary experiments, it was verified that the only available frame rate of the camera (29-30 fps) enables the observation of relevant, recurrent macro phenomena, with enough detail to study the transfer modes. The reader who is more familiar with the GMAW process may find useful to verify the droplet frequency in the results section. It is evident from Figure 2(b) that the vertical distance between the wire tip and the melt pool $\left(y_{f}-y_{m}\right)$ is an upper limit for the size of the droplets, prior to detachment. The work presented by Taguchi (1979a, 1979b) showed that the metal transfer modes 1 to 3 are related to this vertical distance.

\subsection{Deposition}

An interesting addition to the comparison of the conditions that control the transfer modes is to look at different process configurations that result in the same geometry. Consequently, the model reported in Rios (2018) was used to choose two set points that produced very similar layer heights of $1.6 \pm 0.1 \mathrm{~mm}$ and wall widths of $7.5 \pm 0.1 \mathrm{~mm}$, despite having extreme values of current and plasma gas flow rate (PGFR) within the envelope. For each wall, all parameters except current and plasma gas flow rate were kept the same, as indicated in Table 1 and Table 2. One parameter set produced a concave melt pool which exhibited a depression caused by the momentum of the arc gas, while the other produced a convex profile with no depression.

A study was done to determine the lower and upper limits of the wire position $y_{w}$ (Figure 2(a)) in the concave and convex cases. The lower limit was defined by vibration of the wire, which was produced when the wire tip hits the bottom of the melt pool (also called stubbing). The upper limit was defined by the ejection of spatter. From layer six onwards (after steady state thermal conditions are obtained), $y_{w}$ was varied within these limits to observe changes in the metal transfer modes. The time span between layers was between 3 and 5 minutes, to operate the data acquisition systems, including layer height, arc current and voltage and video.

Table 1 PTA experimental variables.

\begin{tabular}{lcc}
\hline & Convex & Concave \\
\hline Current, A & 160 & 133 \\
PGFR, L min & 0.80 & 5.00 \\
\hline
\end{tabular}

Table 2 Constant experimental parameters.

\begin{tabular}{|c|c|}
\hline Parameter & Value \\
\hline $\mathrm{TS}, \mathrm{mm} \mathrm{s}^{-1}$ & 5.0 \\
\hline WFS, $\mathrm{mm} \mathrm{s}^{-1}$ & 50.0 \\
\hline Diffuser Length, mm & 2.4 \\
\hline Diffuser Angle, $^{\circ}$ & 36.8 \\
\hline Electrode Set-back, mm & 2.4 \\
\hline Wire diameter, mm & 1.2 \\
\hline
\end{tabular}

\subsection{Data acquisition and processing}

Arc voltage and current were recorded with a sampling rate of $10 \mathrm{kHz}$. The arc voltage was then filtered with a low pass filter of $200 \mathrm{~Hz}$ and converted with the algorithm presented further down, into a signal composed of only -1 and +1 , for low and high voltage, respectively, which was called the constructed signal. The constructed signal is similar to the one captured by Kobayashi et al. (1996), and was used to calculate the contact frequency (CF) and the relative time for droplet growth (GT), defined by: 


$$
\begin{gathered}
C F=\sum_{1 \mathrm{sec}} \text { Voltage drops } \\
G T=\sum_{1 \mathrm{sec}} \frac{t_{g}}{t_{g}+t_{c}}
\end{gathered}
$$

For each sample in the signal, both indices were calculated over the previous one second window. The contact frequency at any given instant, is simply the count of voltage drops over the previous second. It was found that one second is a suitable compromise between the delay of using a longer time window and the unstable value that results from using a shorter time window. The ignition and extinction of the arc were used to match the video and voltage data sets.

The constructed signal was created with an algorithm, the outputs of which are summarised in Figure 3 for an idealised and actual voltage signal. Consider the sudden rise of $1 \mathrm{~V}$ shown at $20 \times 10^{-4} \mathrm{~s}$ in Figure 3(a). To detect it, the simple moving average (SMA) of the arc voltage, over $n$ samples was used. Then, two different values of SMA were compared to each other (Equation 3):

$$
\Delta t=S M A_{t}-S M A_{t-n}
$$

One at the time of interest $t$ and the other, $n$ samples before $t$, where $n$ corresponds to a certain amount of time and is an adjustable parameter in the algorithm.
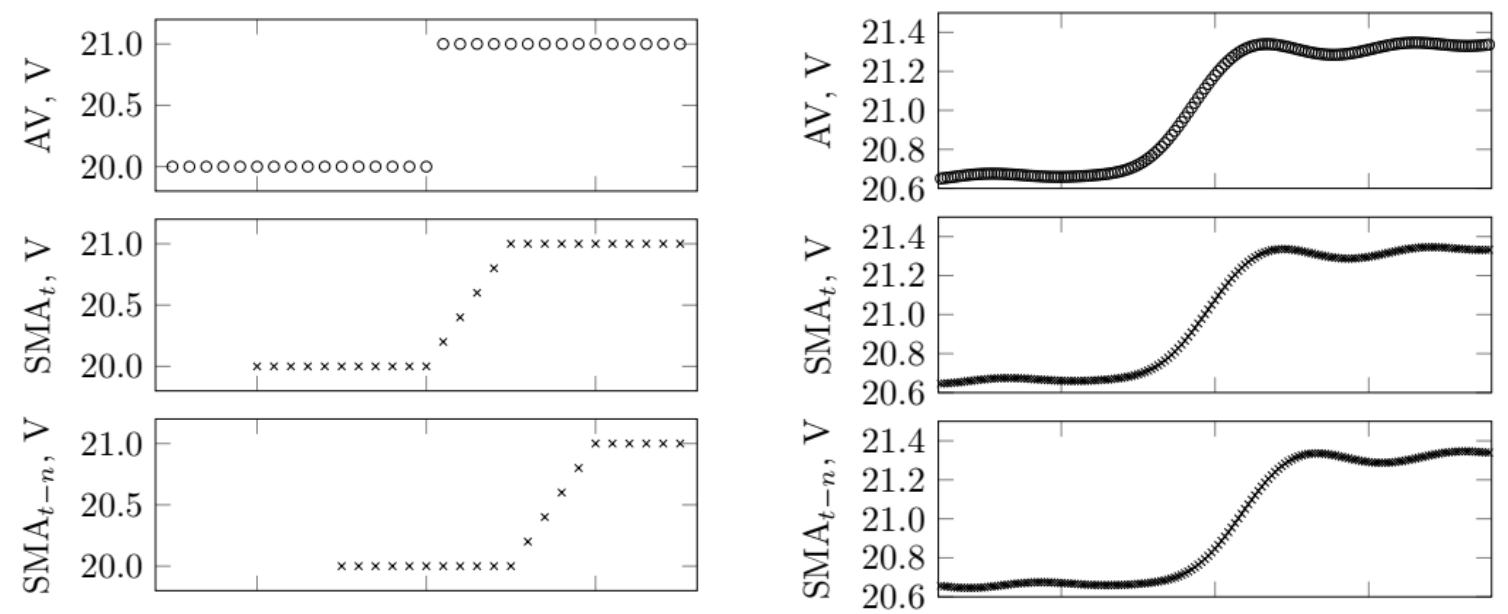

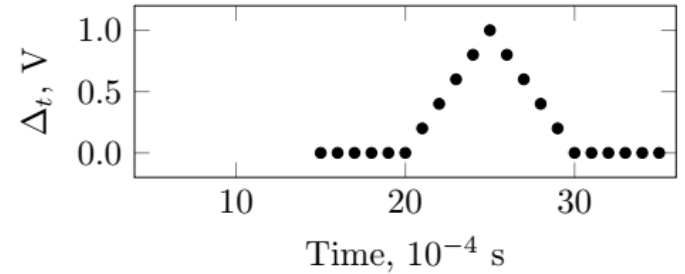

a

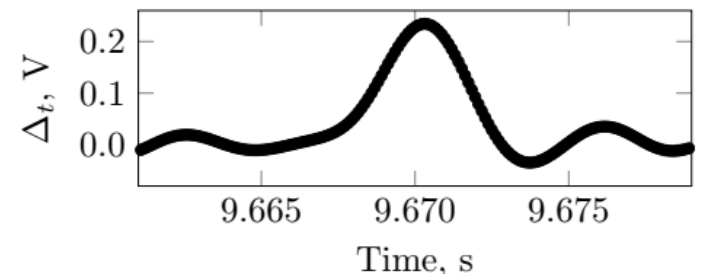

b

Figure 3 Plots of the original arc voltage, and how this is analysed to detect a rise in its value through the use of $\Delta_{t}$ : (a) artificial signal with $n=5$ samples and (b) actual signal with $n=10$ samples.

Their difference $\Delta_{t}$ indicates when a sudden change in arc voltage is observed, when it is above or below a given threshold. The value of this threshold is determined based on historical data and is provided in the results section. The function $\Delta_{t}$, defined in Equation 3 and plotted in Figure 3(a), will have a local minimum or maximum $n$ samples after the point where the sudden change in arc voltage occurred. Decrements in the signal will produce local minima, whereas increments will produce local maxima. Thus, it is possible to build a signal that strongly facilitates further processing, which is the aforementioned constructed signal. 


\section{Results}

\subsection{Arc voltage signal and video recording}

Figure 4 shows a characteristic arc voltage drop, accompanied by video frames, to show the corresponding stages of the transfer of metal from the wire to the melt pool. The images confirm that, as expected, sudden drops in arc voltage occur when the wire touches the melt pool. When droplets electrically connect the wire to the melt pool, they shorten the mean arc length, by changing the shape of the liquid surface. Similarly, there is a sudden rise of arc voltage, as soon as contact between wire and melt pool is lost. These phenomena have been observed in TIG welding multiple times by Sorensen and Eagar (1990) and Yudodibroto et al. (2004).

The arc voltage signal of layer number 6 and above was used to produce the constructed signal, as indicated by the algorithm in subsection 2.4, prior to further analysis of the metal transfer modes. The effectiveness of the algorithm is illustrated in Figure 5, where a couple of extracts of the arc voltage signal (AV) are followed by the corresponding $\Delta_{t}$ values and the constructed signal. The two adjustable parameters in the algorithm were the threshold which was set between $0.14 \mathrm{~V}$ and $0.34 \mathrm{~V}$ and the number of samples over which the average was calculated $(n)$ was between 4 and 10 . The sensitivity of the algorithm is increased by reducing the threshold or the number of samples $n$. However, an over sensitive setting will generate false positives, thus reducing the effectiveness of this tool. Therefore, the robustness of this algorithm depends strongly on the choice of these settings. Nevertheless, in this study it was not possible to analyse all signals with a single choice of these parameters, this remains a matter for further investigation.

Figure 6 presents a comprehensive set of arc voltage samples, for each melt pool type. The individual graphs are ordered from top to bottom, in a way that corresponds to the wire position, so that the graph at the top belongs to the highest position of the wire. Considering the time of contact $\left(t_{c}\right)$ and droplet growth $\left(t_{g}\right)$, the shape of the arc voltage signal reveals an evolution in the metal transfer modes, from flitting falls at an unsteady frequency (TM3), when the wire is at a high position, through to a state of more regular very quick drops (TM2), and then gradually increasing $t_{c}$, until the signal permanently stays low, as the wire dips into the melt pool (TM1), at the lowest position. [ONLY FOR ONLINE VERSION OF THE PAPER: Video files are provided for TM1 (Link_to_Video1.mp4), TM2 (Link_to_Video2.mp4) and TM3 (Link_to_Video3.mp4).]

Figure 7 shows contact frequency vs. growth time index (GT - see equation 1) for the plots presented in Figure 6. Clearly, it is not possible to characterize the shape of the arc voltage signals by means of contact frequency only. However, by following the time of growth index it is easy to group the points in three ranges $\mathrm{GT} \leq 0.2,0.2<\mathrm{GT} \leq 0.8,0.8<\mathrm{GT}$, which are useful to define the transfer modes. The transfer modes in PTA-WAAM are thus defined in terms of the growth time index, within these boundaries. A more representative view of the possible combinations, extracted from all layers above number 5 is shown in Figure 8 for the concave and convex cases. This is, a total of more than 15 minutes of arc monitoring, for more than 20 experimental conditions. Considering that the growth time and the contact frequency can be calculated for every sample, at this sampling rate there are $10^{4}$ values of each index per second. Therefore the plots include only $0.3 \%$ of the available data, which was extracted evenly from the whole data set. Both plots in Figure 8 are similar to those presented by Kobayashi et al. (1996), where the contact frequency has a maximum value above $4 \mathrm{~Hz}$ when growth time is between 0.4 and 0.6. However, there is also a new feature found in this work, which is a pronounced rise of contact frequency at the upper end of the growth time values, labelled zone $\mathrm{D}$. The configurations within zone $D$ correspond to the highest positions of the wire, where the contact frequency is irregular. The contact frequency is particularly high with the concave melt pool and will be discussed later. 


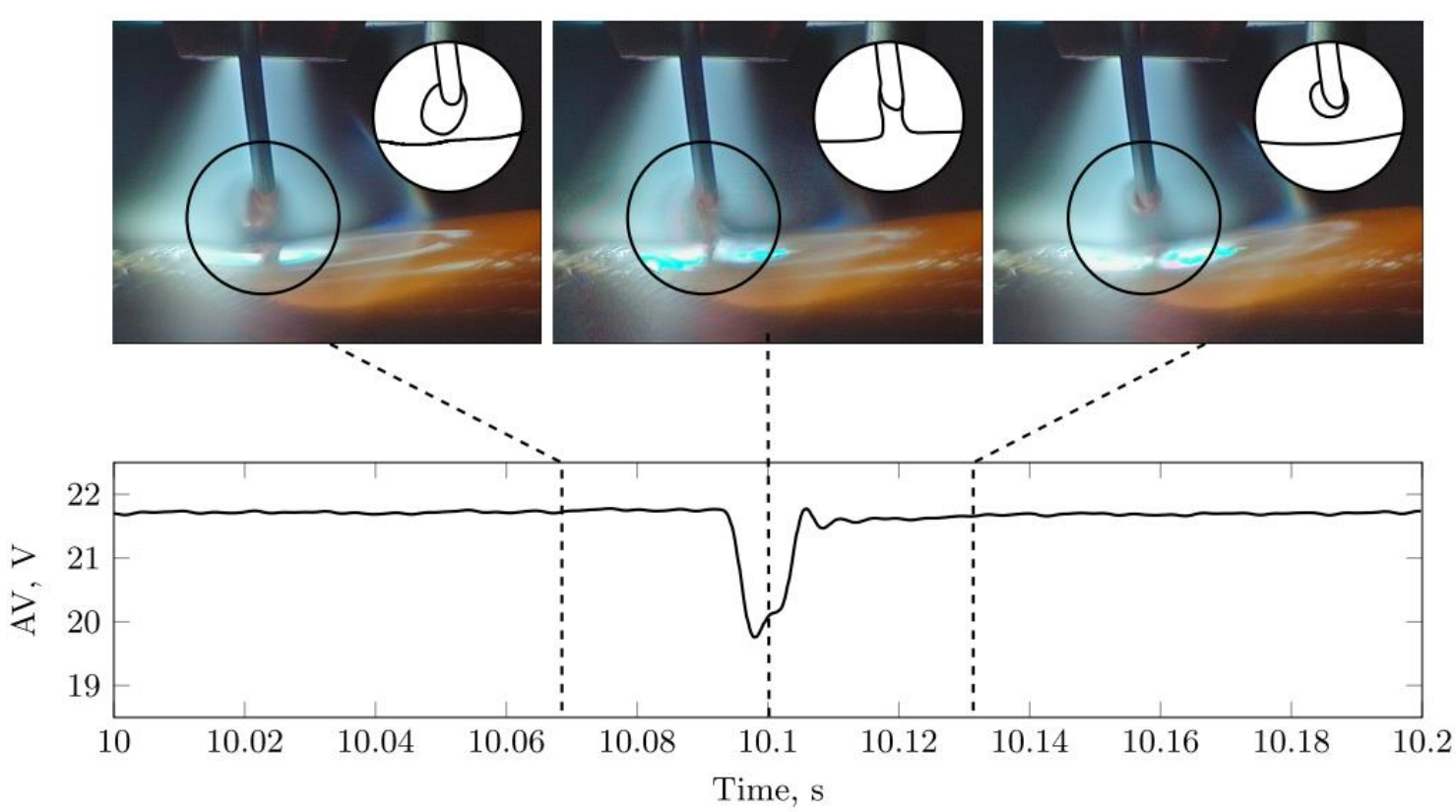

Figure 4 Evidence that arc voltage drops occur when the wire is in contact with the melt pool. The sequence corresponds to the convex weld pool (See Table 1).
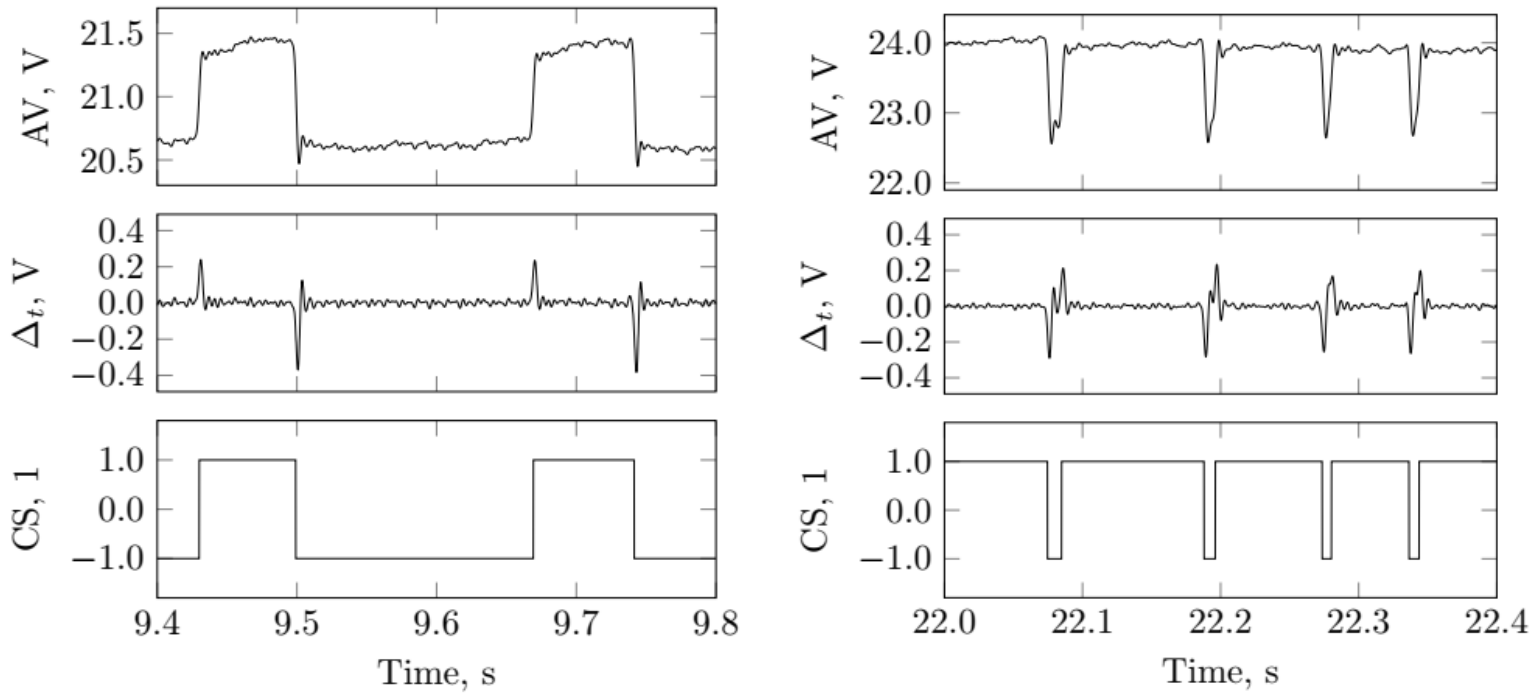

a

$b$

Figure 5 Graphs showing how $\Delta_{t}$ can be used to convert the arc voltage signal (AV) into the constructed signal (CS), which can be more easily processed for (a) TM2 and (b) TM3. 

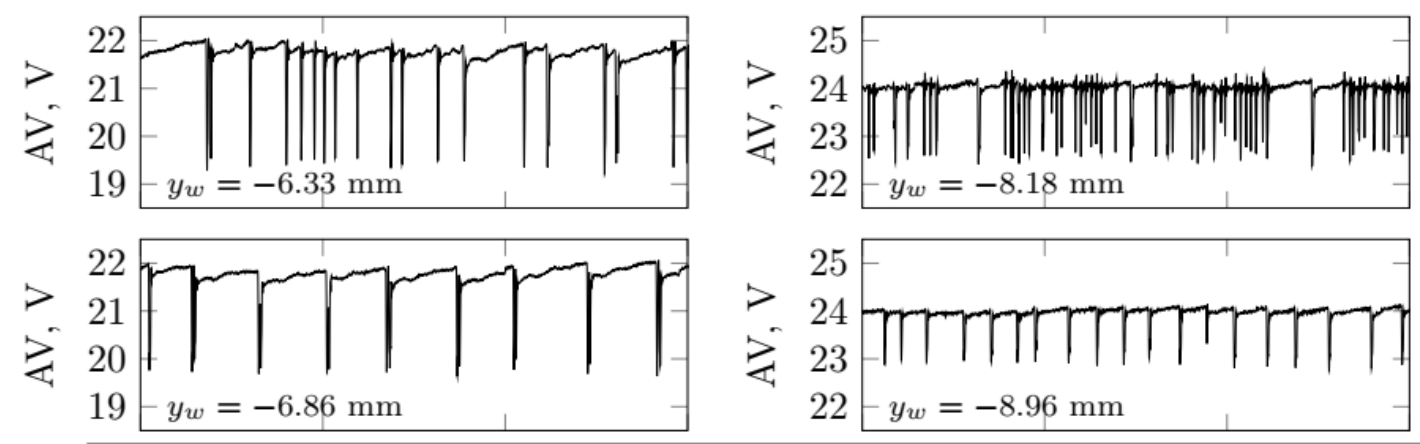

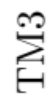
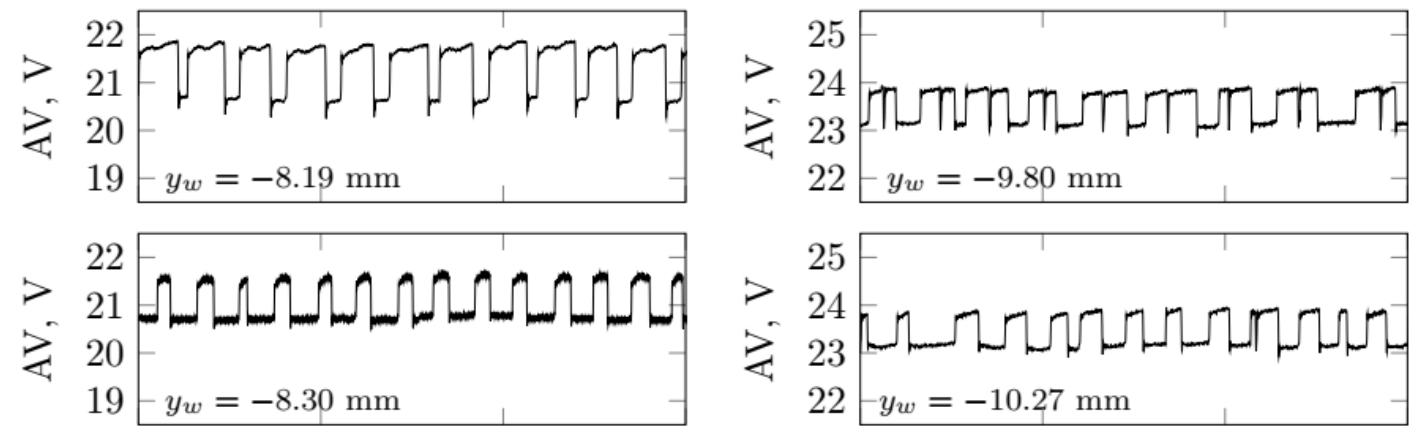

$\sum_{\mathrm{E}}^{\stackrel{Y}{H}}$
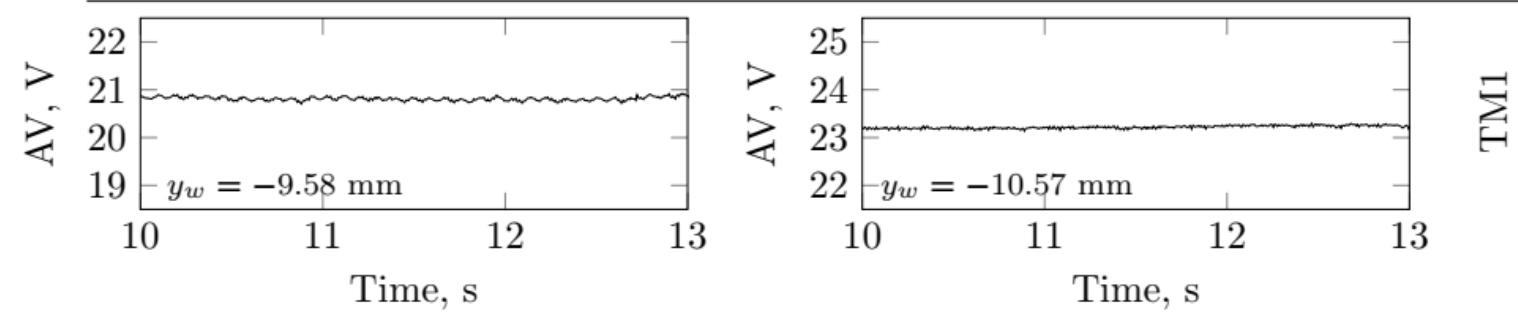

a

b

Figure 6 Evolution of arc voltage for the concave and convex melt pools, relative to wire position for (a) convex and (b) concave melt pools.

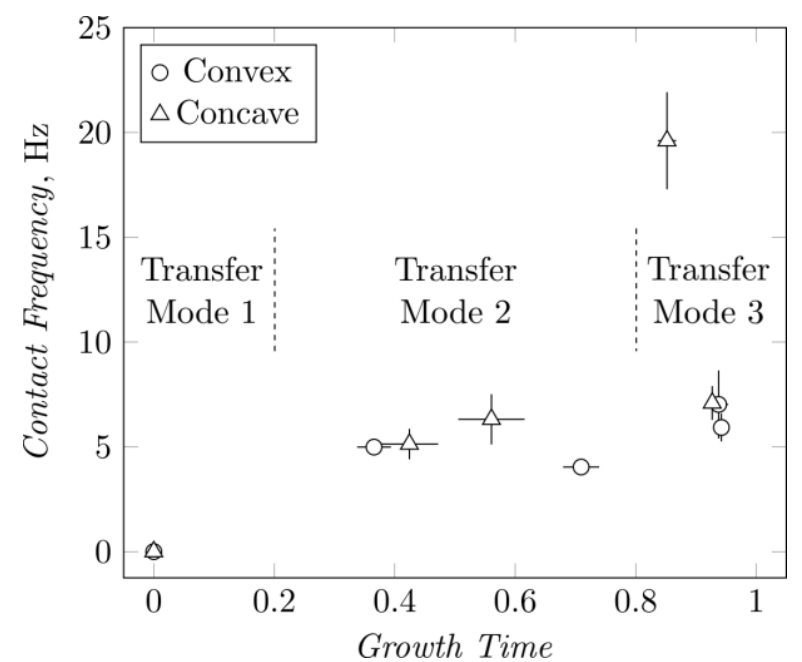

Figure 7 Contact frequency vs. time of growth index for the process conditions presented in Figure 6. 


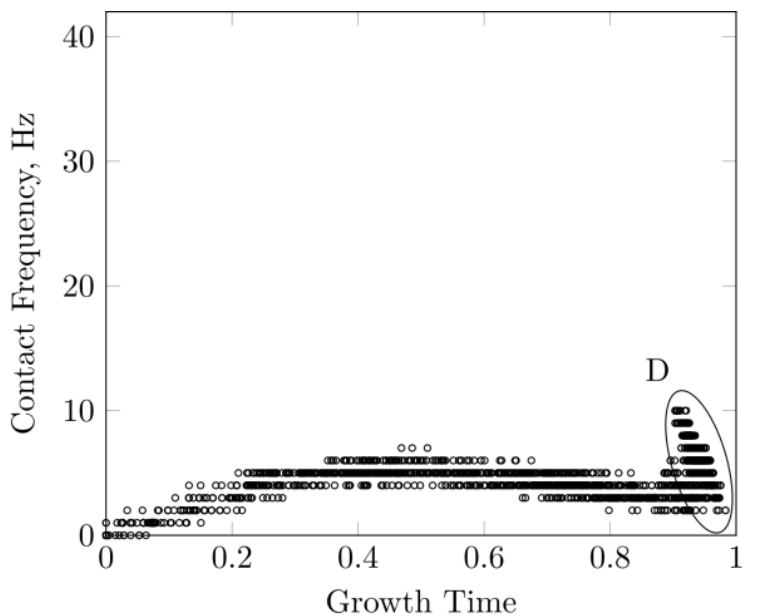

a

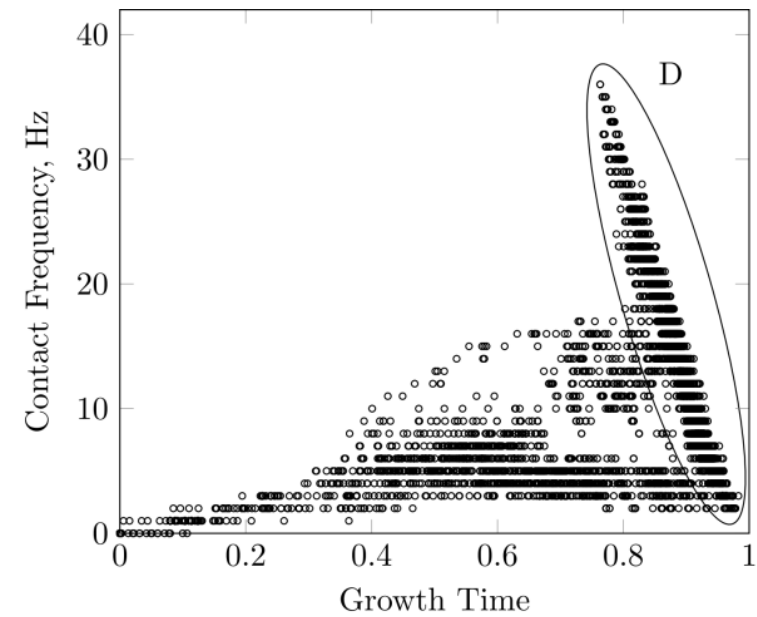

b

Figure 8 Contact frequency vs. time of growth index for (a) convex and (b) concave melt pools.

\subsection{Transfer modes and wire position}

The useful range of $y_{w}$ (Figure 2(a)) was about $4 \mathrm{~mm}$ for both the concave and convex melt pools, using the criteria described in subsection 2.3. Hence, the range of vertical wire positions that can be used with each process condition was similar. Figure 9 shows the relationship between the wire vertical position $y_{w}$ and the growth time index. In both experiments, the growth time index is quite sensitive to wire position and the relationship is not proportional, but closer to a step function. Figure $9(a, b)$ show that in the experiment with a concave melt pool, the transition between TM1 and TM3 occurs at about $y_{w}=-10.2 \mathrm{~mm}$, whereas in the convex case the transition occurs at approximately $y_{w}=$ $-8.7 \mathrm{~mm}$. The relationship between wire position and the melt pool shape was studied by plotting the growth time index versus the wire position relative to the melt pool surface $\left(y_{w}-y_{m}\right)$ in Figure $9(c, d)$. In the concave case, the wire position where the transition occurs is slightly closer to the melt pool surface than in the convex case and therefore larger droplets are produced in the transition in the convex case. 


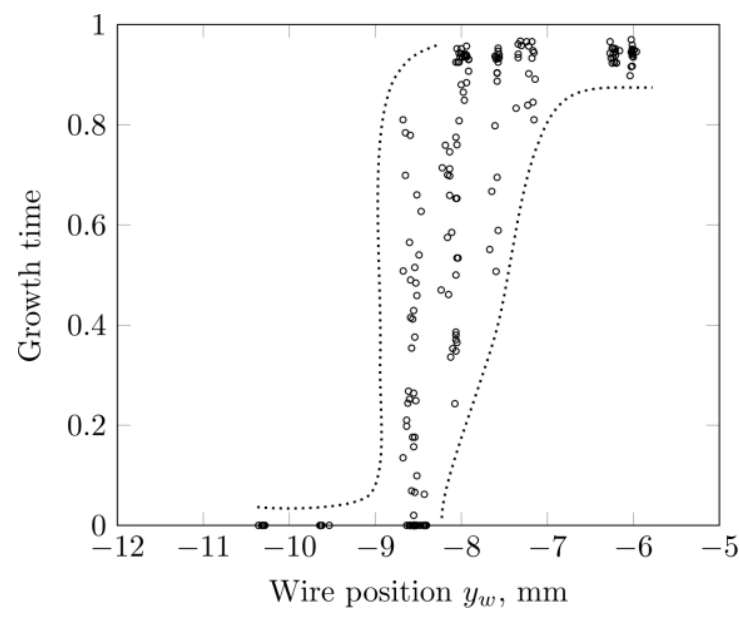

a

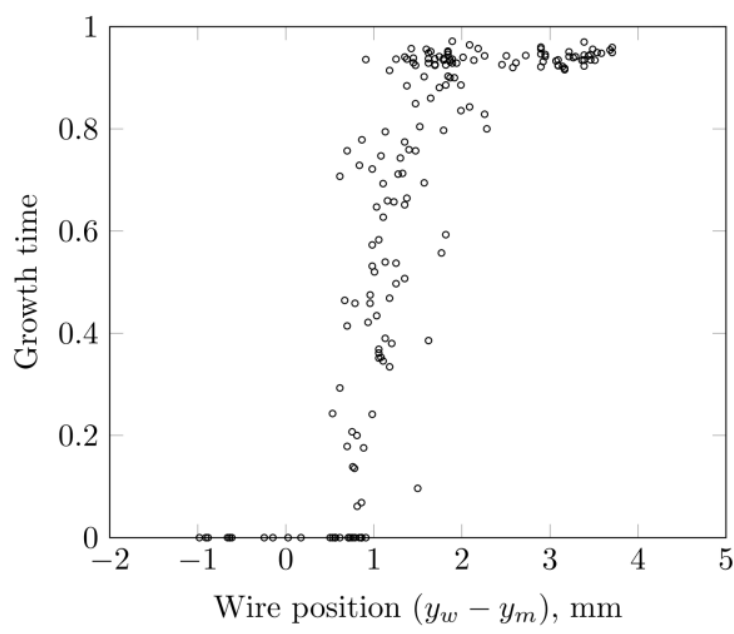

C

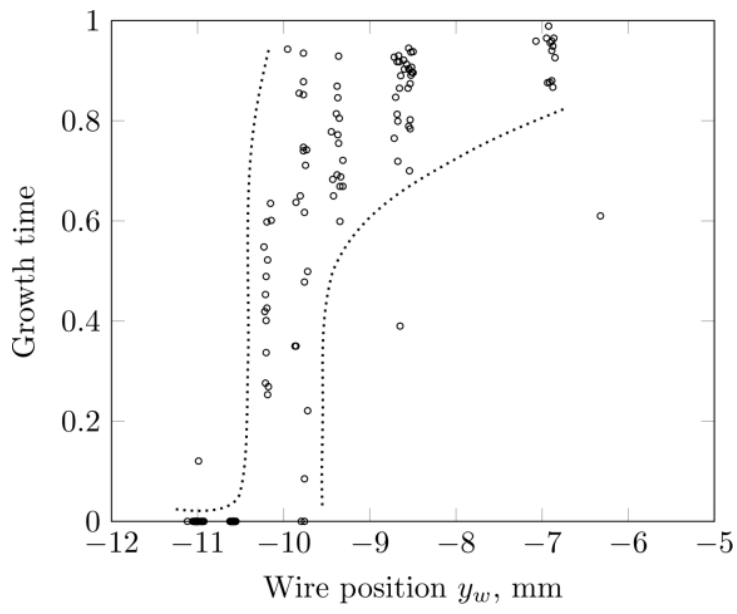

b

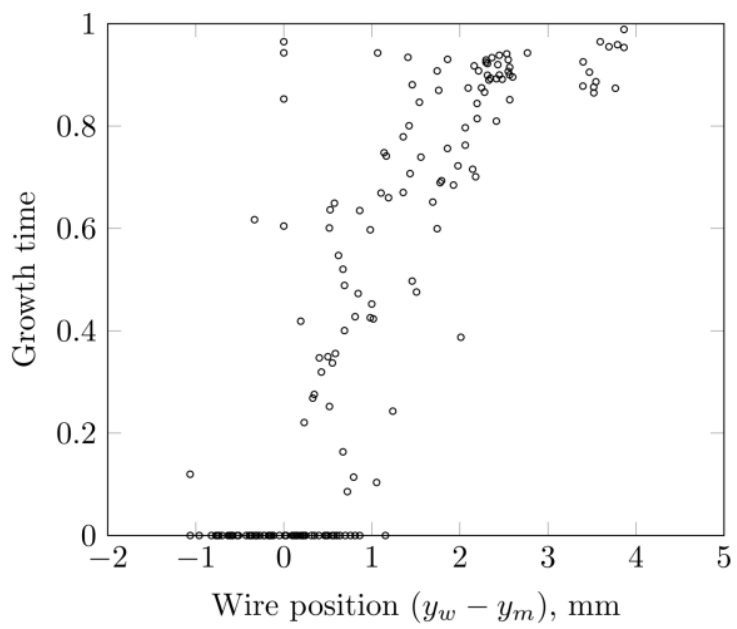

d

Figure 9 Growth time index versus wire position with respect to the nozzle for (a) convex and (b) concave melt pools and the melt pool $\left(y_{w}-y_{m}\right)$ for (c) convex and (d) concave melt pools.

\subsection{Metal transfer mechanisms}

Figure 10 compares the contact and no-contact conditions in TM3, for the convex and the concave cases, and shows how the contact between the wire and melt pool might affect the anode surface shape, which in turn would affect the average arc length and the arc voltage. It is interesting to notice that the concave melt pool presents TM3 with smaller droplets and smaller disturbances, than the convex melt pool. This observation fits with the voltage drops shown in Figure 6, at the second highest $y_{w}$ values of each melt pool. Comparing the magnitude of the voltage drops, the convex melt pool presents a drop of about $2 \mathrm{~V}$, while the concave melt pool presents drops of $1 \mathrm{~V}$. Furthermore, the contact frequency is about $3 \mathrm{~Hz}$ for the convex melt pool, whereas it is more than twice that for the concave melt pool. Careful observation of TM3 in Figure 6 shows that in some cases within the same voltage drop there are two local minima, indicating that at least sometimes large droplets are detached in two contacts with the melt pool. Figure 6 shows that the double minima are more clearly observable at the second highest wire positions of each melt pool. 


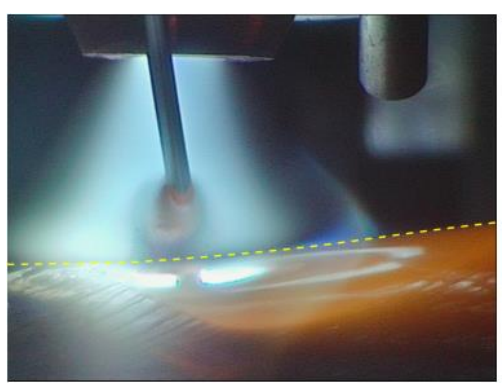

a

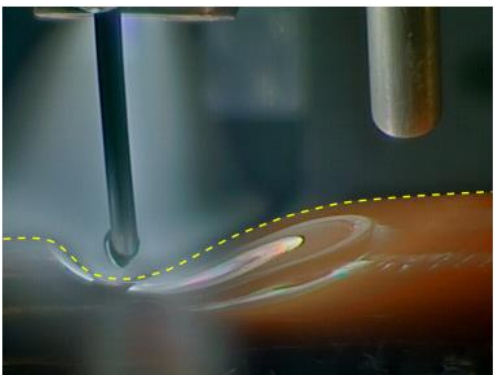

d

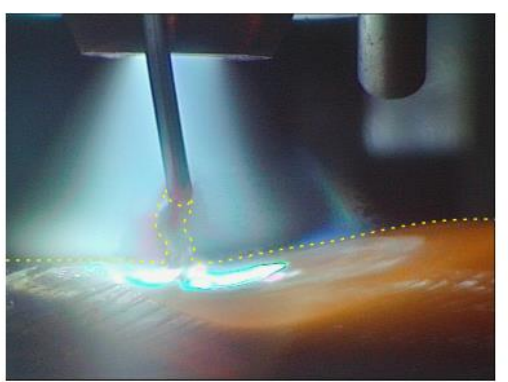

b

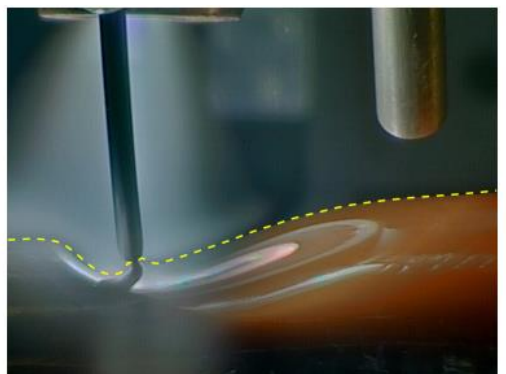

e

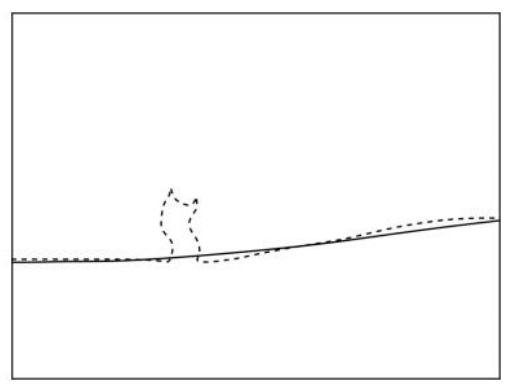

C

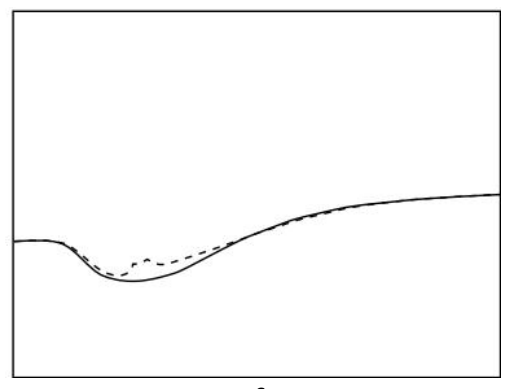

f

Figure 10 The effect of the wire on the shape of the anode surface in TM3, for the convex case (a-c) and the concave case $(d-f) ;(a, d)$ with gap, no contact. $(b, e)$ contact established; and $(c, f)$ overlapped view.

In TM3, as soon as the wire contacts the puddle, the liquid bodies of droplet and melt pool become one, with a waist at the base of the droplet, as shown in Figure 11(a). Figure 11(b) shows that as the waist between the droplet and the melt pool widens, the bulk of the droplet flows into the melt pool, reducing the perimeter at the upper zone of the fluid thread. This condition was filmed by Yudodibroto (2010). This transition is driven by a sudden change in the action of surface tension, which is first holding the droplet against gravity and then, by pinching the liquid bridge, it acts in the same direction as the gravitational force. Large droplets, such as those produced in TM3 with a convex melt pool, produce more intense disturbances on the melt pool surface, because they produce a larger deflection of the surface and transport more mass. Immediately after the thread is broken (Figure 11(c)), the melt pool surface is in an unstable condition. The surface pulls strongly, rapidly displacing large volumes of liquid within the melt pool, as shown by Yudodibroto (2010). In contrast, the disturbance to the melt pool is much smaller in TM1 and contact is re-established more quickly.

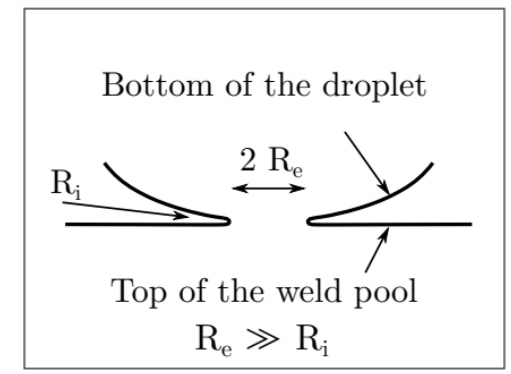

a

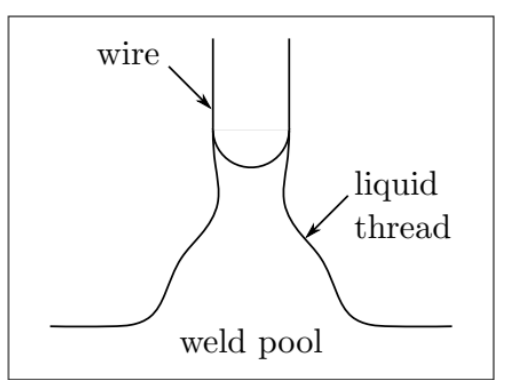

b

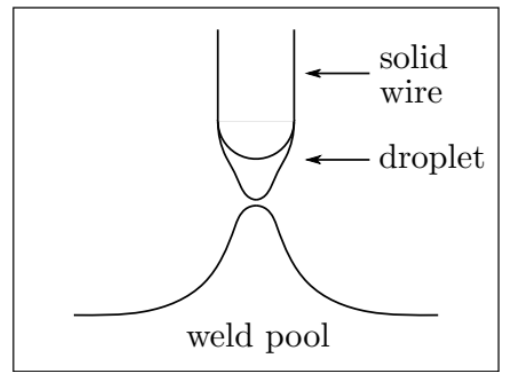

C

Figure 11 Liquid bridge evolution in TM3. (a) Close up of the spot where the droplet meets the melt pool. Notice the principal radii of curvature; (b) a large droplet sinks quickly into the melt pool, pulled down by gravity and squeezed by surface tension; (c) when the liquid bridge brakes apart, the two remaining liquid surfaces have an unstable shape. 


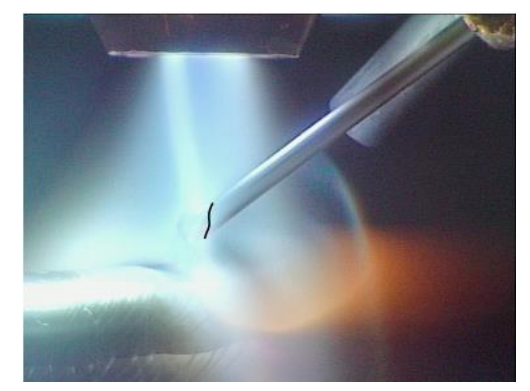

a

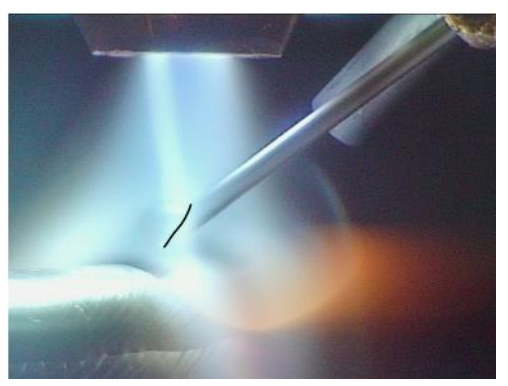

b

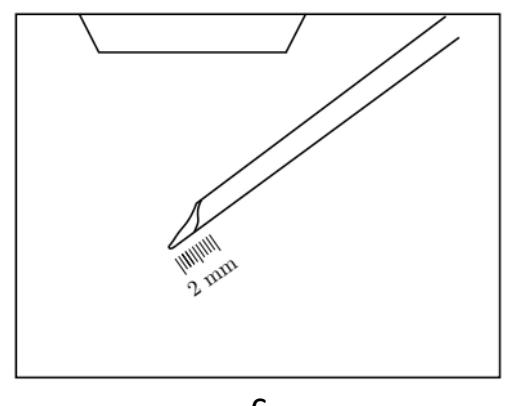

C

Figure 12 (a) Droplet growth and displacement of the wire fusion line in TM2, in the convex weld pool. Note that the camera is placed diagonally in front of the torch; (b) at the beginning of droplet growth, the lower edge of the wire fusion line is at its most upstream position; (c) during droplet growth, the presence of the droplet affects the position of the edge where the wire melts.

TM2 is the most interesting of the three and is shown in Figure 12. In TM2, the space available for droplet growth is only enough for a small droplet, which forms a sustainable fluid thread between the wire and the melt pool. During droplet growth, the droplet shadows the wire tip from the hot gases of the arc, which results in the displacement of the lower part of the wire fusion line downwards, as shown in Figure 12 (b).

Once the thread with the melt pool forms, the thin wedge of solid metal at the wire tip (Figure 12(b)) loses the shielding effect of the droplet and is therefore exposed to the heat of the arc and heat is conducted upwards from the melt pool. Consequently, the wire fusion line is displaced back upwards along the wire. The retraction of the wire fusion line stretches the thread until it cannot sustain the surrounding disturbances and breaks up (Figure 12(a)). After breakup, there is a wave on the melt pool surface. This wave reflects off the back of the melt pool and then raises towards the small droplet that formed in the meantime, which is just the right size to sustain a new thread. A detailed sequence of this wave phenomenon is shown in Figure 13, with a plot of the consecutive positions of the melt pool surface, in Figure 13(e). 


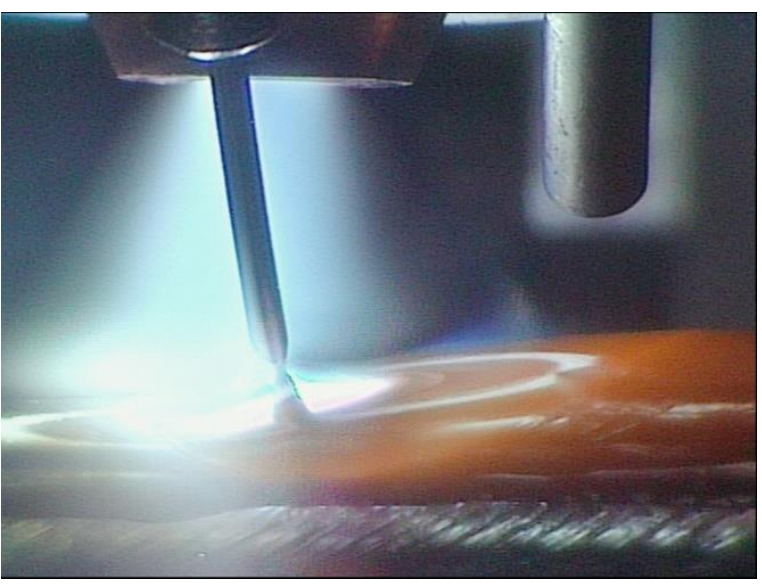

a

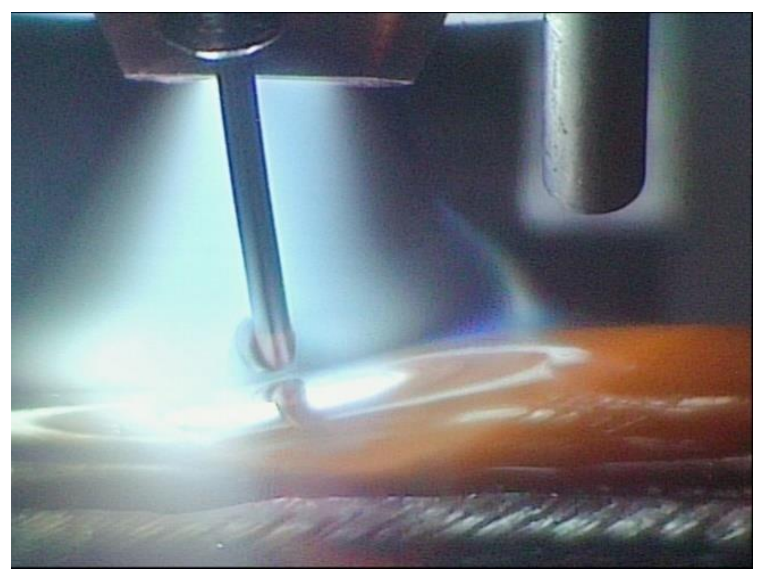

C

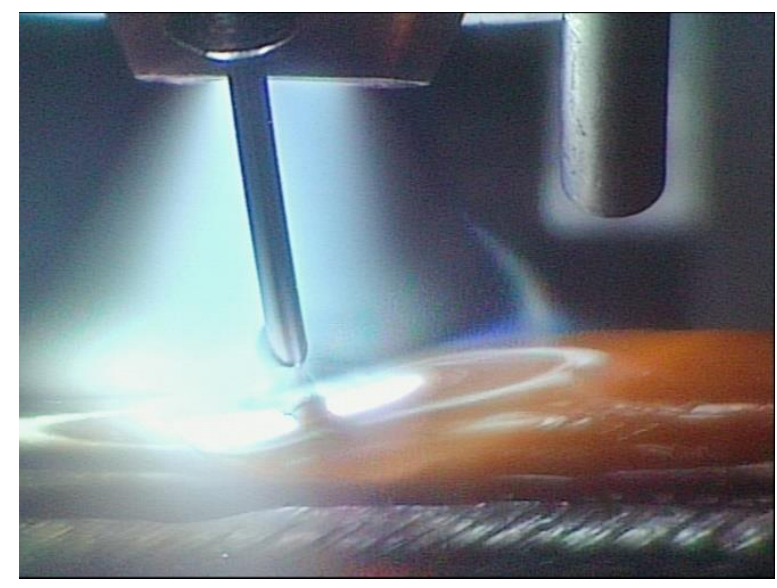

b

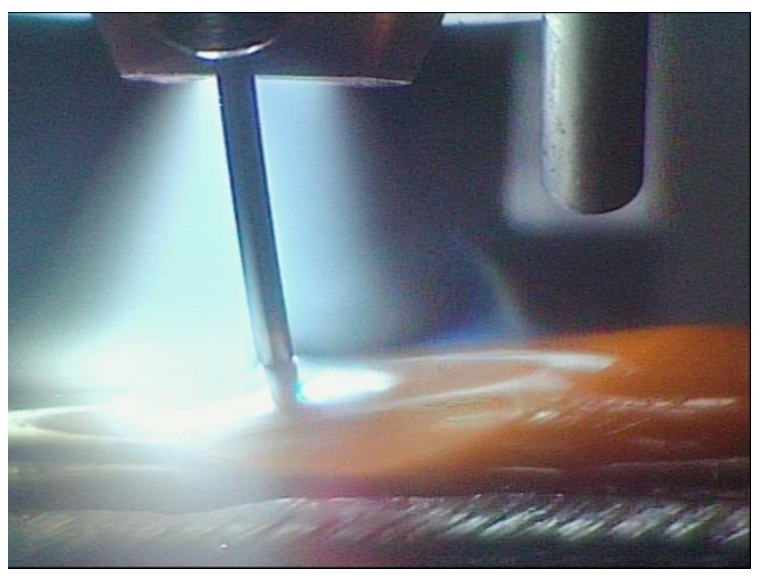

d

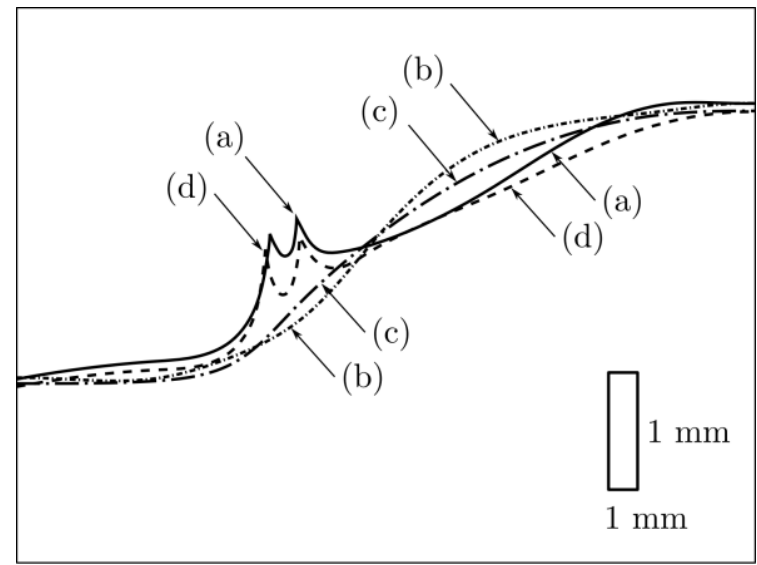

e

Figure 13 (a-d) Four stages of droplet formation and transfer in TM2, in the convex weld pool. (a) Before thread breakup; (b) wave in the back; (c) the wave reflects forward and a new droplet grows; (d) the thread is recovered; (e) the consecutive changes in surface profile, magnified 4 times in the vertical direction.

\section{Discussion}

One of the most interesting observations was the high contact frequencies that were observed with high wire positions with both concave and convex melt pools ( $D$ zones in Figure 8 ). The cause of the erratic behaviour is the drag from the arc, which acts on the droplets while they are still growing and 
attached to the wire tip. However, the droplet size at which this occurs depends on whether a concave or convex melt pool is used. With the convex melt pool, the arc momentum is lower which allows the molten droplets to grow much larger, because it is the weight of the droplet which finally overcomes the action of surface tension at the wire tip. In addition, if the wire position is high the droplets can grow much larger because there is more space available. Large droplets are more vulnerable to drag, because they typically have a larger cross section and the lower ratio of surface to volume reduces the influence of surface tension. Video recording showed large droplets wondering, blown by the arc, rotating violently around the wire tip, which are eventually deposited in the melt pool or become spatter. These large rotating droplets can briefly touch the melt pool multiple times (which results in a brief drop in voltage) before finally being deposited in the melt pool (Figure 6(a)). With the concave melt pool, the plasma gas momentum is much higher and produces regular contact of small droplets with the melt pool at high frequency. Consequently, with the concave melt pool, the range of wire position which enables transfer mode 3 to occur is much wider and the range of transfer mode 1 is decreased.

There are two important practical questions that arise from this work: which melt pool is preferable and which transfer mode is preferable. With respect to the former question, the plots of the growth time index vs wire frequency in Figure 9 show how a much larger range of wire positions are in transfer mode 1 and the transition to transfer mode 3 is relatively abrupt with the convex melt pool. The larger range of wire positions in transfer mode 1 is undoubtedly due to the deeper melt pool; with a concave melt pool, the layer of liquid is thinner because of the depression as described in Mendez and Eagar (2003) and it may eventually freeze, stopping the flow of liquid metal towards the rear of the melt pool, as shown by Meng et al (2012), which limits the range of wire positions that can be used. Transfer mode 3 is the most undesirable transfer mode due to its unstable nature and spatter is significant. A much larger part of the operational range with the concave melt pool operates in transfer mode 3 (see Figure 9) making this melt pool geometry undesirable. Finally, concave melt pools can cause cavities within the deposit if the molten liquid freezes prior the depression collapsing as reported in Martina et al. (2012).

Secondly given that the convex melt pool is desirable, which transfer mode -1 or 2 is most desirable? Both lead to stable metal transfer. With transfer mode 1 the operational range is large, however it's not possible to detect when the wire is approaching the lower position that causes stubbing from the voltage output: an optical technique would be required making the control system more complex. Transfer mode 2 can be more easily detected with voltage signals and the contact frequency could be used to accurately position the wire. Transfer mode 2 exists within a range of wire position of about 2 $\mathrm{mm}$, which could lead to instabilities in the algorithm that controls the height of the wire and or the torch. However, it is unlikely that very fast adjustments in wire or torch position would be required in normal operation. Therefore, both transfer modes have their strengths and weaknesses and on balance transfer mode 2 is perhaps preferable from the perspective that the torch/wire position can be controlled from the voltage output alone. Finally, as stated earlier, laser-wire deposition can be controlled by detecting the increase in resistance of the wire which occurs at the transition between transfer modes 1 and 2. Video imaging from this study was analysed to see whether a similar approach could be used for plasma deposition. In laser-wire deposition, there is no significant drag to pull from the droplets, which possibly allows a larger range of wire feed speed or wire position, to operate in transfer mode 1 . In plasma deposition the arc momentum strongly decreases the stability of the liquid bridge, possibly reducing the applicability of this resistance-based method.

Finally, as stated in the introduction, previous researchers have used continuity of the wire with the melt pool to control the position of the torch, which requires an additional electrical circuit. The advantage of the method presented in this work is that no such circuit is required, and it only requires monitoring of the arc voltage which would be done anyway. The difference is subtle, and both can be successfully implemented, so the preferred method may largely be the preference of the system integrator who develops the WAAM equipment. 


\section{Conclusions}

The analysis presented above highlights the following findings:

- The arc voltage drops in PTA-WAAM, when the wire and the melt pool are in contact due to the shortening of the arc column. This can be used to detect how the wire is transferred into the melt pool which could be useful for developing a control system.

- The contact time can vary from a few milliseconds in TM3 to a continuous condition in TM1.

- The range of available wire positions is about the same for both concave and convex melt pools and similar to those found in literature $\left(\Delta \mathrm{y}_{\mathrm{w}} \approx 4 \mathrm{~mm}\right)$.

- High plasma gas flow-rates produce a melt pool with a large depression and increase the likelihood of TM3 making these processing conditions undesirable. The aerodynamic drag tears the droplets away from the wire, turning them into spatter; reduces the droplet size, and increases the contact frequency. This can be detrimental to the stability of the process, and the shallower melt pool is more prone to forming cavities.

- The wire position at which the transition between TM1 and TM3 occurs is strongly dependent on the position of the melt pool surface and occurs over a relatively narrow range of wire positions.

- Convex melt pools are desirable for a production system, and both TM1 and TM2 provide stable deposition. The operational range of TM1 is greater, however the position of the wire cannot be easily detected from arc voltage alone. TM2 can be detected with the arc voltage and for this reason is slightly preferred for a control system that avoids optical methods.

\section{Acknowledgements}

The research presented above was funded partially by the National Commission for Scientific and Technological Research CONICYT, from the Chilean Ministry of Education, through the Doctoral scholarship program; and by the WAAM-Mat research program of Cranfield University.

\section{References}

Dilthey, U., Fuest, D., Scheller, W., 1995. Laser welding with filler wire. Opt. Quantum Electron. 27, 1181-1191. doi:10.1007/BF00326474

Hagqvist, P., Heralić, A., Christiansson, A.-K., Lennartson, B., 2014. Resistance measurements for control of laser metal wire deposition. Opt. Lasers Eng. 54, 62-67. doi:10.1016/j.optlaseng.2013.10.010

Heralić, A., Christiansson, A.-K., Lennartson, B., 2012. Height control of laser metal-wire deposition based on iterative learning control and 3D scanning. Opt. Lasers Eng. 50, 1230-1241. doi:10.1016/J.OPTLASENG.2012.03.016

Imanaga, S., Haneda, M., Shibata, N., Kobayashi, M., Hino, E., 2000. Development of torch position control and welding condition control technology for all-position, multi-layer GTA welding. Development of fully automatic GTA welding system for pipes (2nd Report). Weld. Int. 14, 356364. doi:10.1080/09507110009549194

Kobayashi, M., Imanaga, S., Haneda, M., Minakuchi, K., 1996. Development of Wire Height Control Technique for All Position GTA Welding -Development of Fully Automated GTA Welding System for Pipe (Report 1). J. Japan Weld. Soc. 14, 546-550.

Martina, F., Mehnen, J., Williams, S.W., Colegrove, P., Wang, F., 2012. Investigation of the benefits of plasma deposition for the additive layer manufacture of Ti-6Al-4V. J. Mater. Process. Technol. $212,1377-1386$.

Mendez, P.F., Mendez, P.F., Eagar, T.W., Eagar, T.W., 2003. Penetration and Defect Formation in HighCurrent Arc Welding. Weld. J. 82, 296S-306S. 
Meng, X., Qin, G. and Zengda, Z. 2016. Investigation of Humping Defect in High Speed Gas Tungsten Arc Welding by Numerical Modelling. Materials and Design 94:69-78.

Miranda, R.M., Lopes, G., Quintino, L., Rodrigues, J.P., Williams, S., 2008. Rapid prototyping with high power fiber lasers. Mater. Des. 29, 2072-2075. doi:10.1016/j.matdes.2008.03.030

Rios, S., Colegrove, P.A., Martina, F., Williams, S.W., 2018. Analytical Process Model for Wire + Arc Additive Manufacture. Addit. Manuf.

Seufzer, W.J., Taminger, K.M., 2007. Control methods for the electron beam free form fabrication process, in: 18th Solid Freeform Fabrication Symposium, SFF 2007. pp. 13-21.

Sorensen, C.D., Eagar, T.T.W., 1990. Measurement of Oscillations in Partially Penetrated Weld Pools Through Spectral Analysis. J. Dyn. Syst. Meas. Control 112, 463-468. doi:10.1115/1.2896166

Taguchi, H., Haneda, M., Imanaga, S., Itsuhiko, S., 1979a. Metal Transfer in Plasma Arc Plasma Arc Welding with Filler Wire Addition ( Report 1 ). Japan Weld. Soc. 48, 488-493.

Taguchi, H., Haneda, M., Imanaga, S., Sejima, I., 1979b. Characteristics of Plasma Arc Welding with Arc Wire Addition (Report 2). Japan Weld. Soc. 48, 1065-1069.

Williams, S.W., Martina, F., Addison, A.C., Ding, J., Pardal, G., Colegrove, P., 2016. Wire + Arc Additive Manufacturing. Mater. Sci. Technol. 32, 641-647. doi:10.1179/1743284715Y.0000000073

Yudodibroto, B.Y.B., Hermans, M.J.M., Hirata, Y., den Ouden, G., 2004. Influence of filler wire addition on weld pool oscillation during gas tungsten arc welding. Sci. Technol. Weld. Join. 9, 163-168. doi:10.1179/136217104225012274

Yudodibroto, R.B.Y.B., 2010. Liquid Metal Oscillation and Arc Behaviour during Welding. Delft University of Technology. 
2018-08-31

Metal transfer modes in plasma pÿWire + Arc additive manufacture

Ríos, Sergio

Elsevier

pÿRíos S, Colegrove P, Williams S. (2019) Metal transfer modes in plasma Wire + Arc additive manufacture, Journal of Materials Processing Technology, Volume 264, February 2018, pp. 45-54 https://doi.org/10.1016/j.jmatprotec.2018.08.043

Downloaded from Cranfield Library Services E-Repository 